\title{
Innovations in Body Force Modeling of Transonic Compressor Blade Rows
}

\author{
David J. Hill $(\mathbb{D}$ and Jeffrey J. Defoe \\ Department of Mechanical, Automotive, and Materials Engineering, 401 Sunset Ave, Windsor, ON, Canada N9B 3P4 \\ Correspondence should be addressed to Jeffrey J. Defoe; jdefoe@uwindsor.ca
}

Received 4 March 2018; Revised 17 May 2018; Accepted 7 June 2018; Published 9 July 2018

Academic Editor: Ryoichi Samuel Amano

Copyright (C) 2018 David J. Hill and Jeffrey J. Defoe. This is an open access article distributed under the Creative Commons Attribution License, which permits unrestricted use, distribution, and reproduction in any medium, provided the original work is properly cited.

\begin{abstract}
Aeroengine fans and compressors increasingly operate subject to inlet distortion in the transonic flow regime. In this paper, innovations to low-order numerical modeling of fans and compressors via volumetric source terms (body forces) are presented. The approach builds upon past work to accommodate any axial fan/compressor geometry and ensures accurate work input and efficiency prediction across a range of flow coefficients. In particular, the efficiency drop-off near choke is captured. The model for a particular blade row is calibrated using data from single-passage bladed computations. Compared to full-wheel unsteady computations which include the fan/compressor blades, the source term model approach can reduce computational cost by at least two orders of magnitude through a combination of reducing grid resolution and, critically, eliminating the need for a time-resolved approach. The approach is applied to NASA stage 67. For uniform flow, at $90 \%$ corrected speed and peak-efficiency, the body force model is able to predict the total-to-total pressure rise coefficient of the stage to within $1.43 \%$ and the isentropic efficiency to within $0.03 \%$. With a $120^{\circ}$ sector of reduced inlet total pressure, distortion transfer through the machine is well-captured and the associated efficiency penalty predicted with less than $2.7 \%$ error.
\end{abstract}

\section{Introduction}

In the preliminary design of compressors, maintaining low computational cost for numerical simulation of the blade aerodynamics is paramount. Traditionally, various $1 \mathrm{D}$ and $2 \mathrm{D}$ mathematical models have been employed. 1D calculations (mean-line codes) yield the shortest computational time but with a reduction in both accuracy and available flow details. 2D models (streamline curvature methods, throughflow methods) provide a greater degree of accuracy; however, the flow is typically assumed to be axisymmetric.

In this paper, a 3D distributed source term modeling approach, commonly and henceforth referred to as a body force model, is developed. The approach can be applied to any axial compressor and captures efficiency drop-off near choke. This model is used to capture distortion transfer and efficiency penalties associated with inflow distortion. A body force model replaces physical blades in a numerical simulation with a volumetric source term field. Doing so considerably reduces the grid density required to capture key phenomena such as loading distribution, overall pressure rise, and work input when compared to bladed unsteady Reynolds-averaged Navier-Stokes (URANS) computations. More significantly, the source term model is a "smearedout" circumferential average of the flow field; even in the case of nonaxisymmetric inflow, source terms are timeinvariant, eliminating the need for an unsteady calculation. In circumferentially nonuniform inflow, there is no frame of reference in which the flow is steady. However, the smearedout forces are not frame-dependent and do not vary with time if two conditions are met regarding the flow [1]: the flow nonuniformity must have a characteristic length scale significantly larger than the blade pitch and the local rotor reduced frequency must be sufficiently small, $g_{\text {red }} \ll 1$. The local rotor reduced frequency is expressed as

$$
g_{\text {red }}=\frac{c_{x} / V_{x}}{2 \pi / \omega},
$$

where $c_{x}$ is the axial chord, $V_{x}$ is the axial velocity, and $\omega$ is the compressor rotational speed. Thus, the body force 
TABLE 1: Design characteristics for NASA stage 67 rotor at $90 \%$ speed and maximum efficiency [10].

\begin{tabular}{lclc}
\hline Parameter & Value & Parameter & Value \\
\hline corrected angular speed $\Omega(\mathrm{rad} / \mathrm{s})$ & 1512 & hub solidity $\sigma_{\text {hub }}$ & 3.11 \\
$M_{\text {rel, tip }}$ & 1.20 & tip solidity $\sigma_{\text {tip }}$ & 1.29 \\
total-to-total pressure ratio $p_{t 2} / p_{t 1}$ & 1.48 & $\left(r_{\text {hub }} / r_{\text {tip }}\right)_{\text {inlet }}$ & 0.375 \\
corrected mass flow rate $\dot{m}(\mathrm{~kg} / \mathrm{s})$ & 31.10 & $\left(r_{\text {hub }} / r_{\text {tip }}\right)_{\text {outlet }}$ & 0.478 \\
number of rotor blades $B$ & 22 & maximum isentropic efficiency $\eta_{\text {is }}(\%)$ & 92.2 \\
aspect ratio $A R$ & 1.56 & tip clearance $/ r_{\text {tip }}$ & 0.39 \\
\hline
\end{tabular}

model is able to capture the response of a turbomachine to nonuniform inflow using only a steady calculation.

The distributed source term approach has been extensively used in the literature to study fan and compressor response to inlet flow distortion. A few recent examples which do not form an exhaustive list include the following: (1) Defoe and Spakovszky investigated the generation and propagation of fan rotor shock noise due to inlet swirl distortion [2, 3], (2) Thollet et al. used body force modeling to reduce the computational cost in studying intake-fan interaction [4], (3) Defoe et al. examined low-speed boundary-layer-ingesting fan and compressor performance using a nonaxisymmetric throughflow method [1], and (4) Guo and $\mathrm{Hu}$ observed multistage compressor performance in the presence of inlet flow distortion [5]. Most of these papers employed different approaches for obtaining the body force model used; what follows is a brief overview of approaches relevant to those adopted and built upon in the current paper.

The methodology was first introduced by Marble [6], replacing a physical blade row with an infinite number of infinitely thin blades. In studying short-wavelength stall inception and distortion transfer in multistage compressors, Gong was able to expand upon Marble's approach by enabling the source terms to respond to local flow properties [7]. Volumetric source terms themselves are broken down in to a normal force per unit mass, $f_{n}$, and parallel force per unit mass, $f_{p}$. The normal component acts perpendicular to the relative streamlines, acting to reduce the deviation of the flow from the blade camber surface. The parallel component acts against the stream-wise direction, thus generating viscous losses through the blade row.

Based on an adaptation from Gong's distributed source term model, Peters developed a body force model to investigate fan inlet and nacelle design parameters for low pressure ratio fans with increased fan and inlet coupling [8]. Peters' model includes a radial component in the normal force, accounting for blade lean and radial streamline shifts. The normal force is dependent on, among other variables, a calibrated blade loading coefficient, $K_{n}$. The calibration process requires nonzero blade metal angle and nonzero flow deviation. Peters implemented an off-design formulation to capture the variation in blade losses with operating condition [8]. Peters' formulation uses a quadratic dependence on mass-averaged relative Mach number at the blade row inlet, combined with a quadratic dependence on local relative velocity inherited from Gong. This formulation produces loss buckets typical of turbomachines.

More recently, an incompressible, inviscid, normal force model was developed by Hall, Greitzer, and Tan [9]. The normal force model is a function of local flow quantities and blade camber angle. Different from previous body force methods, it can be formulated without the need of calibration data (experimental or single-passage RANS computations). This is helpful in preliminary design as full blade profiles need not be developed. The normal force model is developed such that blade rows of low solidity produce a normal force equivalent to thin airfoil lift theory $\left(c_{l}=2 \pi \delta\right)$. Conversely, in high solidity blades, the blade-to-blade relative flow is unable to deviate from the blade metal angle.

In this paper, an innovative methodology for calibrating a body force model of a transonic compressor is demonstrated. A compressible adaptation of the incompressibleflow normal force model developed by Hall et al. [9] is developed in conjunction with an innovation to Peters' loss model which enhances the ability to capture differences in efficiency fall-off as the flow coefficient is either increased or decreased from the maximum efficiency point. The model is shown to accurately capture overall and local machine performance in both uniform and nonuniform inflow, onand off-design. A reduction in computational cost of at least two orders of magnitude is obtained when compared against traditional full-annulus, time-resolved computations. To assess model performance, the compressor performance metrics and flow field details are compared against previously published experimental data as well as full-annulus, bladed computations.

\section{Approach}

2.1. Transonic Compressor Studied. Computations in this work are carried out on a transonic compressor, NASA stage 67 [11]. This single-stage axial compressor has a mean hub-totip radius ratio of 0.427 , so significant radial flow streamline shifts are possible. The spinner nose is stationary; only a portion of the hub in the vicinity of the rotor rotates. All computations are carried out at $90 \%$ of the design corrected rotational speed, yielding a tip relative Mach number of 1.20 at peak-efficiency. Additional design characteristics for the rotor at $90 \%$ corrected speed are given in Table 1 and for the stator in Table 2. 
TABLE 2: Design characteristics for NASA stage 67 stator at $90 \%$ speed and maximum efficiency.

\begin{tabular}{lcll}
\hline Parameter & Value & Parameter & \\
\hline number of stator blades $B$ & 36 & tip solidity $\sigma_{\text {tip }}$ & Value \\
aspect ratio $A R$ & 2.16 & $\left(r_{\text {hub }} / r_{\text {tip }}\right)_{\text {inlet }}$ & 1.33 \\
hub solidity $\sigma_{\text {hub }}$ & 2.57 & $\left(r_{\text {hub }} / r_{\text {tip }}\right)_{\text {outlet }}$ & 0.50 \\
\hline
\end{tabular}

2.2. Body Force Modeling Approach. The normal force model in this work is based on the incompressible, inviscid turning force model developed by Hall et al. [9]:

$$
f_{n}=\frac{\delta B}{2 r\left|n_{\theta}\right|} W^{2}
$$

where $\delta$ is the flow deviation angle, $B$ is the number of blades, $W$ is the velocity magnitude in the rotational frame of reference moving with the blade, $r$ is the streamtube radius, and $\left|n_{\theta}\right|$ is the circumferential projection of the camber surface normal. To adapt this model for transonic flow, a compressibility correction field, $\epsilon$, is added to the local flow deviation angle:

$$
f_{n}=\frac{(\delta+\epsilon) B}{2 r\left|n_{\theta}\right|} W^{2},
$$

where $\epsilon$ is a function of axial and radial location within each blade row's swept volume. This field is calibrated to enforce the correct relative flow angle throughout the blade rows; the calibration is machine-specific and not meant to be an empirical constant for all turbomachines. The calibration procedure for $\epsilon$ imposes no restrictions on the type of turbomachine that can be modeled, as will be detailed in Section 2.4. Within a blade row, it is not possible to produce both the correct swirl velocity and relative flow angle simultaneously due to the absence of blade metal blockage. This phenomenon is captured in Figure 1; a mismatched chord-wise axial velocity profile dictates that the body force model can only produce one of the two aforementioned properties accurately. The consequence of this is incorrect rotor work input since the trailing edge deviation is underpredicted. This follows directly from the Euler turbine equation:

$$
h_{t 2}-h_{t 1}=\omega\left(r_{2} v_{\theta 2}-r_{1} v_{\theta 1}\right)
$$

where $h_{t}$ is the total enthalpy and $v_{\theta}$ is the swirl velocity in a stationary frame of reference. For adiabatic flow, the total enthalpy change is governed purely by work done on the fluid. To produce the correct total enthalpy rise in the body force model, the change in swirl velocity must match actual machine behavior. This is accomplished through a twopart calibration: the compressibility correction factor tuning described earlier and effective camber reduction. Based on work by Patel et al. [12], the camber is reduced linearly from leading edge to trailing edge:

$$
\Delta \kappa(x, r)=\Lambda \frac{\left(x-x_{L E}(r)\right)}{\left(x_{T E}(r)-x_{L E}(r)\right)}\left(\beta_{T E}(r)-\beta_{L E}(r)\right),
$$

where $\Delta \kappa$ is the effective camber reduction of the rotor, $\Lambda$ is a blade row specific constant, $x$ is the axial coordinate, and $\beta$ is the local relative flow angle. To further simplify the model, the effects of blade lean are neglected. Whereas, in Hall's model, the normal force is perpendicular to the relative stream-wise direction in a plane shared by the blade normal $\widehat{n}$ and the local relative velocity $W$, the force is instead taken to be normal to the relative stream-wise direction, purely in the $x-\theta$ plane $\left(f_{n, r}=0\right)$. Within the normal force calculation, $\left|n_{\theta}\right|$ is replaced by $\cos (\kappa)$ to accomplish this. The implications of this simplification are later discussed. The normal force takes the final form

$$
f_{n}=\frac{(\delta+\Delta \kappa+\epsilon) B}{2 r \cos (\kappa)} W^{2} .
$$

The formulation allows for the correct flow angle and thus correct loading at the blade leading edge, as well as correct swirl velocity and hence total enthalpy rise at the blade trailing edge. Ensuring the correct blade loading at the leading edge is critical for the model to be able to drive the upstream flow redistribution in nonuniform flow.

Peters developed a loss model for studying the effect of ultrashort nacelles on low pressure ratio fans [8], which included a quadratic dependence on blade-inlet massaveraged relative Mach number, $\bar{M}_{r e l}^{M}$. This formulation is used to capture increased entropy generation at off-design flow conditions. The low pressure fans studied operate at flow coefficients far from choke where Peters' model was sufficient to produce the desired isentropic efficiency versus flow coefficient characteristic. The flow coefficient is defined:

$$
\phi=\frac{\dot{m}}{\bar{\rho}_{0}^{M} A_{0} U_{\text {mid }}},
$$

where $\dot{m}$ is the mass flow rate, $\bar{\rho}_{0}^{M}$ is the mass-averaged stagnation density, $A_{0}$ is the reference area at domain inlet, and $U_{\text {mid }}$ is the midspan blade speed. Peters' loss model is

$$
f_{p}=\frac{K_{p 1}}{b}\left[\left(\bar{M}_{r e l}^{M}\right)^{2}+K_{p 2}\left(\bar{M}_{r e l}^{M}-M_{r e f}\right)^{2}\right] W^{2} .
$$

$\bar{M}_{\text {rel }}^{M}$ is calculated on a plane of constant axial coordinate at the leading edge of the blade; this is denoted as station 1 for the rotor and station 2 for the stator. The coefficients $K_{p 1}, K_{p 2}$, and $M_{r e f}$ are used to set the peak-efficiency and the slope of the efficiency curve. In Peters' work, $M_{\text {ref }}$ is set to be $\bar{M}_{r e l}^{M}$ at maximum efficiency. Finally, $b$ is the staggered blade spacing,

$$
b=\frac{2 \pi r \sqrt{\sigma} \cos (\kappa)}{B}
$$

where $\sigma$ is the blade solidity. This staggered blade spacing is the distance between two discrete blades in the direction 


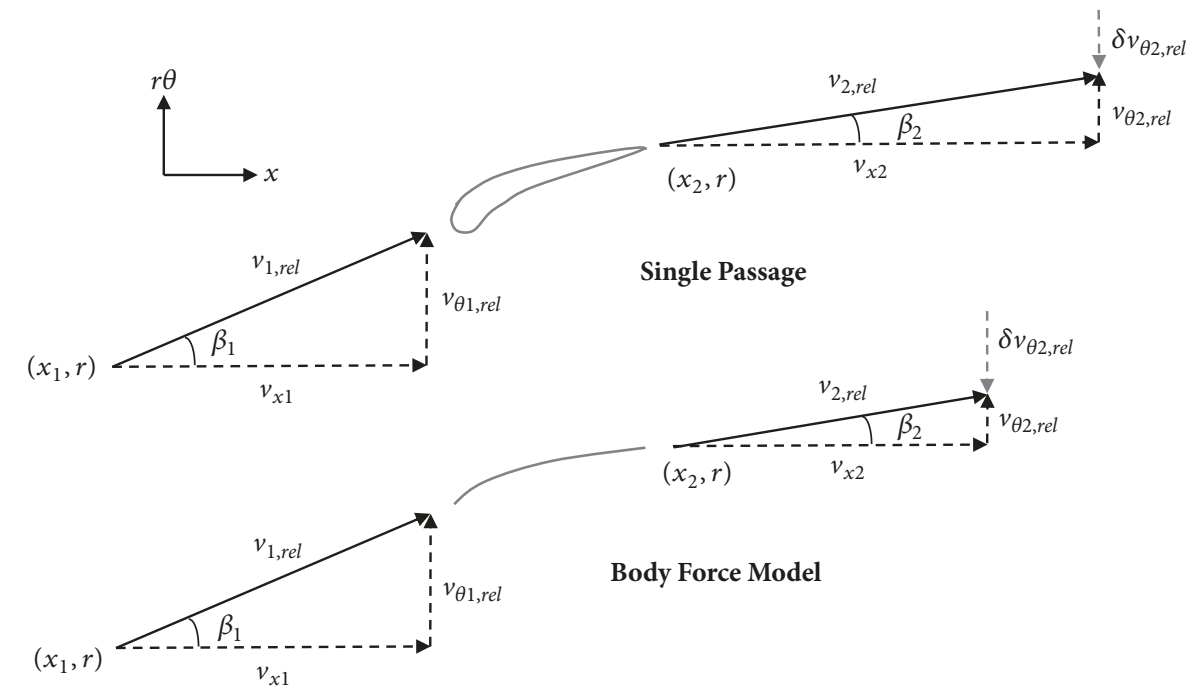

FIGURE 1: Body force model constrained relative flow angle creates a mismatch of relative swirl velocity due to the absence of blade metal blockage.

normal to the camber surface. Observation of (8) clearly illustrates that these coefficients set the value of peak-efficiency or minimum value of $f_{p} b / W^{2}$. The deficiency of this model is its inability to capture the severe drop-off in performance as the machine approaches choke. The efficiency characteristic slope is significantly different at flow coefficients above and below peak-efficiency, in particular for transonic machines where peak-efficiency and choking values of $\phi$ are not far apart. To account for this, two innovations are implemented: $M_{\text {ref }}$ is shifted away from peak-efficiency point, and a piecewise loss function is established:

$$
\begin{aligned}
& f_{p, \text { new }} \\
& =\left\{\begin{array}{ll}
f_{p} & \text { if } \bar{M}_{r e l}^{M}<M_{r e f}^{\prime} \\
f_{p}\left[1+K_{p 2}^{\prime}\left(M_{r e f}^{\prime}-\bar{M}_{r e l}^{M}\right)^{2}\right] & \text { if } \bar{M}_{r e l}^{M}>M_{r e f}^{\prime}
\end{array},\right.
\end{aligned}
$$

where $K_{p 2}^{\prime}$ is a constant used to alter the efficiency at flow coefficients where $\bar{M}_{r e l}^{M}>M_{r e f}^{\prime} . M_{\text {ref }}$ and $M_{\text {ref }}^{\prime}$ serve as local loss minima within their respective multiplicative terms, allowing for two independent "shaping" variables. The values for $K_{p 1}, K_{p 2}, K_{p 2}^{\prime}, M_{r e f}$, and $M_{r e f}^{\prime}$ are calculated iteratively by comparing the resultant efficiency characteristic to the desired efficiency characteristic from high-fidelity computations or experimental data. Each variable is adjusted independently until the desired output is obtained.

2.3. Calibration Data. For the calibration of the body force model, single-passage bladed RANS calculations as well as previously published experimental data by Fidalgo et al. [10] are used. The geometry for the rotor blades was obtained from open-source rotor data [11] and the stator blade geometry was provided by Ewan J. Gunn (Whittle Laboratory, University of Cambridge, $1 \mathrm{JJ}$ Thomson Avenue, Cambridge CB3 0DY, UK) and Vincente J. Fidalgo (Turbomachinery Laboratory, University of Notre Dame, 1165 Franklin Street, Suite 200, South Bend, IN 46601, USA). The duct is extended approximately five rotor axial chords upstream of the rotor and five stator axial chords downstream of the stator. The computations are carried out using ANSYS CFX 17.0 [13]. A combination of Pointwise [14] (upstream and downstream ducts) and TurboGrid [15] (rotor and stator blade passages) was used to create the grid. At the inlet, uniform total temperature, total pressure, and axial flow are specified; the static pressure is set at the domain outlet to yield the desired flow coefficient. The shear-stress transport (SST) turbulence model [16] is used and a wall $y^{+}$value less than 40 is maintained throughout the domain. This includes a maximum value of 10 along the upstream and downstream end-walls. $y^{+}$along the stator blade is kept under 20 and for the rotor blade is largely kept under 20 other than in the tip gap region due to the locally high velocities. At $90 \%$ rotational speed, the flow is generally attached, such that the SST model behaves as the $k-\Omega$ model, where $y^{+}$is far below the recommended maximum of 300 [13]. Additional cell refinement is deemed unnecessary; increased grid density would minimally change the resultant viscous losses, whereas the importance of this work is body force modeling approach development. The details of the near-wall flow features are therefore not critical. The approach ensures that the efficiency and its variation with flow coefficient are well matched between body force and single-passage computations, regardless of the accuracy of the single-passage calibration data. Total grid count for this single-passage calculation is 3.6 million cells. Initial grid sizing was based on a study performed by Fidalgo et al. [10] on the same NASA 67 machine and a grid independence study was carried out to assess the impact of further refinement. The final grid selected was the second of four grids tested. Grid independence was assumed once the rotor pressure ratio and isentropic efficiency changes were each less than $1 \%$. 


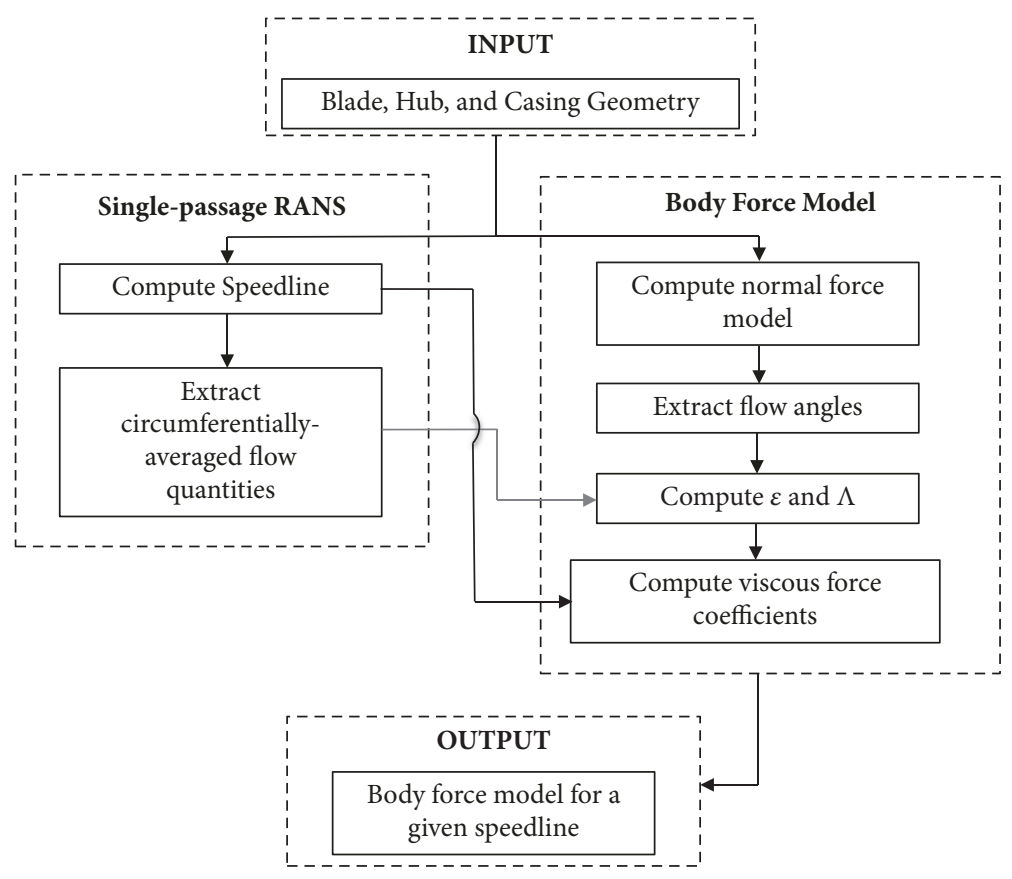

Figure 2: A high-level overview of the distributed source term model calibration process.

As discussed in Section 2.2, only peak-efficiency singlepassage results are required for calibration of the normal force model. For the calibration of the parallel force coefficients, data is required over a range of flow coefficients. In this paper, experimental data provided by Fidalgo et al. [10] is used as a calibration source to reduce the number of single-passage computations required. Within the rotor, the experimental total pressure ratio and isentropic efficiency at various flow coefficients are inputs to the parallel force coefficient calibration process. The body force loss model coefficients are calculated with the objective of matching these properties as closely as possible. In the stator, singlepassage computations are needed to tune the loss model as no stator-alone experimental data is available. Thus, the stator body force model coefficients are calculated on the basis of matching the entropy-based loss coefficient to the singlepassage results.

2.4. Calibration of the Body Force Model. A high-level overview of the body force model calibration process is illustrated in Figure 2. The machine and operating speed of interest must first be selected, from which the singlepassage RANS speedline is calculated. Using both the singlepassage results and, in the case of the machine studied here, experimental data, the normal force model is calibrated $(\epsilon(x, r)$ and $\Lambda$ are determined). Following this, the parallel force model is calibrated simultaneously above and below $M_{\text {ref }}^{\prime}$ by tuning each of the loss model coefficients.

Calculating the $\epsilon$ field requires calibrating against singlepassage RANS data. Initially, this field constant is set to zero. An iterative process is required to calculate the final $\epsilon$ field. The calibration constant is

$$
\epsilon(x, r)=\sum_{i=1}^{n} \epsilon_{i}(x, r),
$$

where $\epsilon_{i}$ is the compressibility calibration field obtained at each iteration and $n$ is the total number of iterations. Within each iteration, $\epsilon_{i}$ is calculated:

$$
\epsilon_{i}(x, r)=\beta_{S P}(x, r)-\beta_{B F}(x, r),
$$

where SP and BF represent single passage and body force, respectively. Each calculation is done concurrently in the rotor and stator. Calibration is complete once (11) produces a change less than $1 \%$ at each location in the entire $\epsilon$ field between iterations, for both blade rows. This iterative formulation enforces the body force to accurately predict the turning no matter the machine. The number of iterations required is expected to rise as flow compressibility and blade metal blockage increase.

The next step is to calculate the camber reduction, $\Lambda$. To do so, the value is altered until the rotor-exit mass-averaged total temperature ratio is best matched with the reference values at peak-efficiency flow coefficient.

The last step is to calibrate the parallel force model. To reduce the computational cost, educated guesses for the initial values of the model constants are important. Given that the flow coefficient at peak-efficiency is known from experimental data, a simplification to (8) is made by combining the coefficients into $\widehat{K}_{p}$ :

$$
f_{p}=\widehat{K}_{p} \frac{W^{2}}{b}
$$

Using this simplified form of the viscous model, $\widehat{K}_{p}$ is determined using the same method employed by Peters [8] at a single flow coefficient (peak-efficiency). From here, the viscous force field at peak-efficiency remains invariant. Thus there is a constraint on the model constants such that the total 


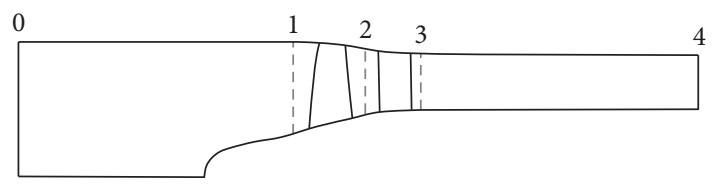

FIGURE 3: Meridional view of the body force computational domain with axial measurement stations.

value of $f_{p}$ at peak-efficiency must be conserved. Previously used values for $K_{p 1}(0.0336)$ and $K_{p 2}(0.6321)$ in the work by Patel [12] served as initial guesses. $M_{\text {ref }}$ is initially set to be equal to $\bar{M}_{r e l}^{M}$ at peak-efficiency as in Peters [8]. The revised parallel force formulation is used in the rotor, and Peters' parallel force formulation is used unaltered in the stator. In the stator, the flow is further from choke, so that Peters' loss model is sufficient. Without computational resources as a limiting factor, the revised parallel force model could be used in both blade rows but significant improvements in accuracy would not be expected.

2.5. Body Force Computational Domain. The body force grid in this work is a $22.5^{\circ}$ slice of the full-annulus machine, created using Pointwise. The axial extents of the domain are identical to the single-passage grid. The rotor and stator blade regions encompass the swept volume of their respective blade rows. The structured grid is constructed on the meridional plane and subsequently revolved. The result is an axisymmetric grid, and uniform inflow calculation results are independent of the circumferential extent. The blade row models are implemented through the use of source terms in the momentum and energy equations within CFX. This is accomplished through a series of expressions and user functions. The forces are frame-independent, allowing for the use of a continuous grid with no mixing planes or interfaces of any kind. A meridional view of the body force domain and axial measurement locations is given in Figure 3. The boundary conditions are set in an identical manner to those in the single-passage computations.

NASA stage 67 contains sharp hub radius changes in the rotor and stator. Within the stator, these area contractions coincide with the adverse pressure gradient produced by flow straightening and end-wall boundary layer development. Due to these conditions, nonphysical severe flow separation was observed. The separation is not consistent with the results observed in the single-passage model. To avoid this flow separation, the no-slip condition is not enforced on end-walls in the body force computations. Within the rotor and stator blade regions, the parallel force model accounts for the endwall losses. Using slip-wall conditions leads to the absence of boundary layer development upstream of the rotor. While it is desired to be able to capture this effect, the use of slip-walls only affects the flow in the bottom 5\% span and upper 5\% span. For the purpose of this study, the overall performance trends are still adequately captured.

Without the physical presence of blades in the body force model, the pressure surface to suction surface flow over the rotor blade tips is not captured. Having no source terms

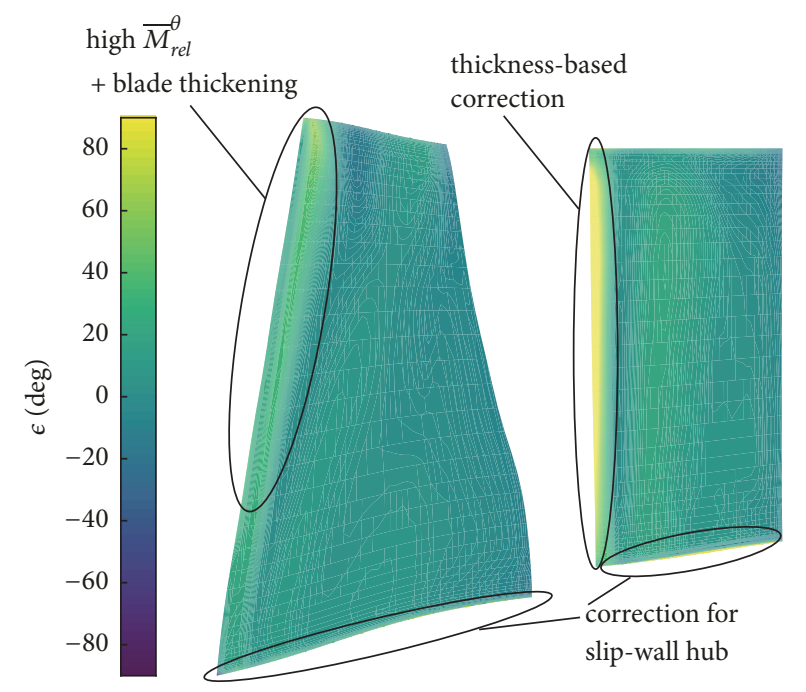

Figure 4: Final $\epsilon$ fields in the rotor (left) and stator (right).

in the tip gap region in the distributed source term model was attempted, but, without physical blades, nonphysical backflow occurs. Thus, in this work, blade forces are extended into the tip gap, which for this machine is less than $0.4 \%$ of the blade span, yielding a tip region swept volume that is only $0.64 \%$ of the entire swept volume of the rotor. A consequence of this approach is that work is done on the flow in the tip gap, meaning the total enthalpy rise is overestimated, and the tip leakage flow details cannot be observed. However, the relatively small tip gap regions mean that the model is still able to predict the overall stage work to an acceptable degree, as will be shown.

A series of five grids were tested for the body force model, with increasing refinement in the axial, radial, and circumferential directions. Grid independence was assumed as the rotor work at peak-efficiency changed significantly less than $1 \%$ between each of the successive grids. Rotor efficiency is not a concern in determining grid independence as the parallel force model is calibrated based on the selected grid. Instead, the additional constraint placed on grid selection is the trade-off between refinement and computational cost. As grid density increases, work input by the rotor changes at a rate that is acceptable, $<0.5 \%$ between all cases. The efficiency, however, is largely dependent on the grid discretization. The selected grid has a full-wheel cell count of $4.48 \times 10^{6}$ cells, corresponding to the second level of the five grid densities tested. For comparison, this grid contains a cell count reduction of over 95\% relative to the bladed RANS computational domain. The grid contains 60 cells in the spanwise direction, 50 cells in the axial direction for each blade row, and 0.71 cells/degree in the circumferential direction.

\section{Results}

3.1. Resultant Body Force Model. The $\epsilon$ field converged after 19 iterations and the resultant spatial distribution for NASA stage 67 is depicted in Figure 4. It is evident that a larger compressibility correction is required at increasing span 


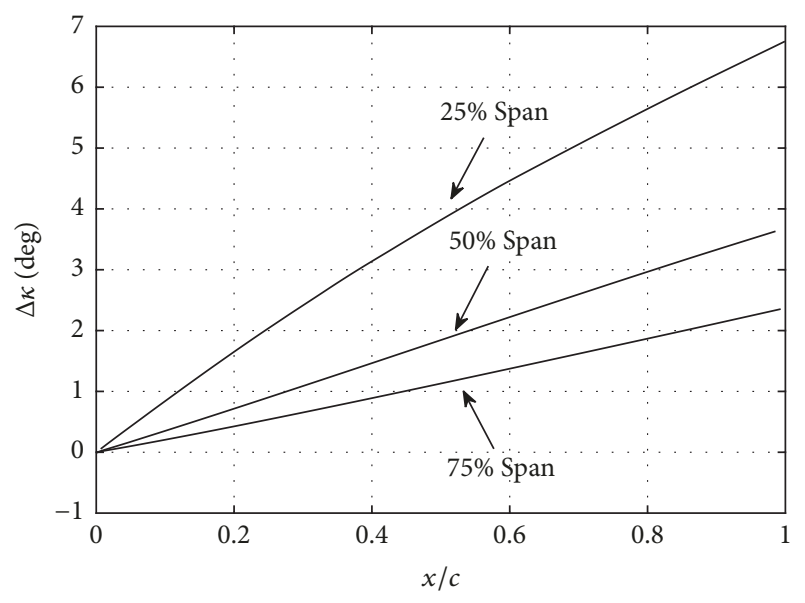

FIGURE 5: Normal force model rotor camber reduction.

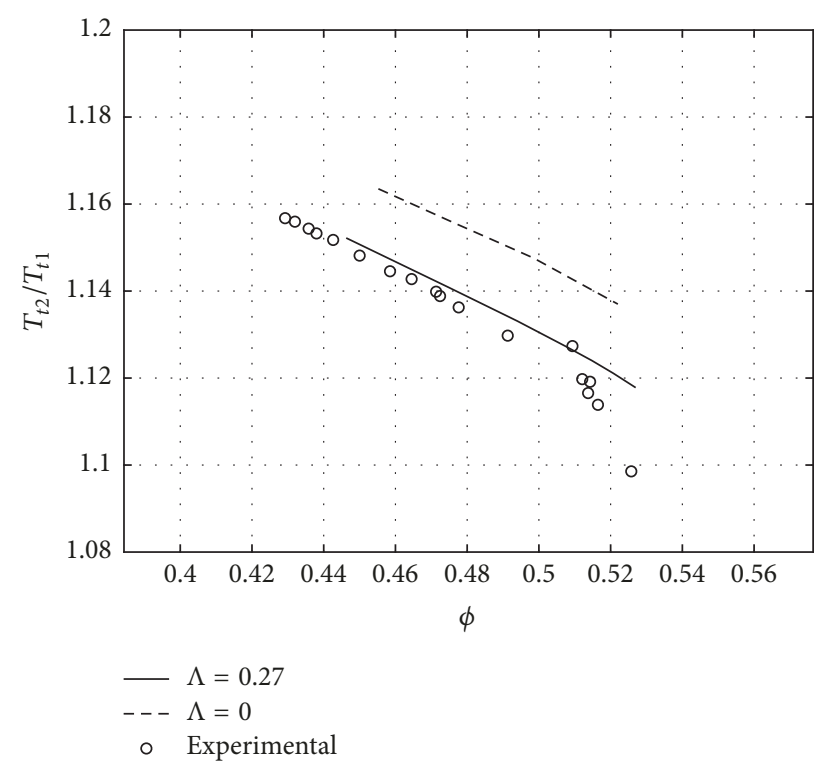

FIGURE 6: Rotor work input characteristic before and after blade recambering, $90 \%$ rotational speed.

values in the rotor and along the stator leading edge. This is expected, as in the rotor, at increasing radius, there is an associated increase in relative Mach number while in the stator the swirl and thus the Mach number are highest around the leading edge. Additionally, the compressibility correction is largest where the blade thickness gradient is largest. In the rotor, the correction factor field closely resembles the relative velocity field. In the stator near the hub, the correction is large to match the flow angles in the no-slip end-wall singlepassage calculations.

After seven iterations, the camber reduction constant converged to a value of $\Lambda=0.27$. The resultant $\Delta \kappa$ for the rotor is shown in Figure 5 and the effects of camber reduction are shown in Figure 6; the work input by the rotor is shifted downwards to match machine performance. Important to note is that the process of camber reduction does not appreciably alter the characteristic slope. In incompressible

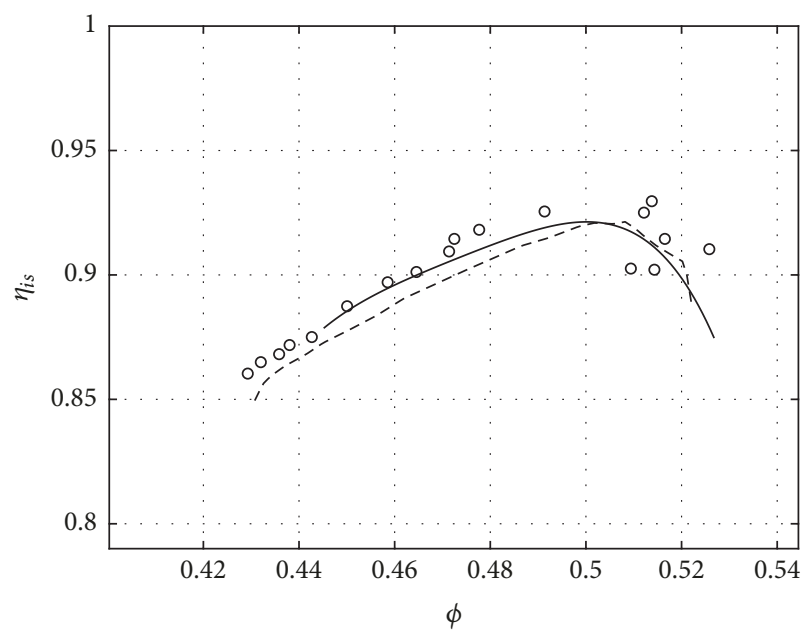

$$
\begin{array}{ll}
\text { - } & \text { Experimental } \\
--- & \text { URANS } \\
- & \text { Body Force }
\end{array}
$$

FIGURE 7: Body force model rotor isentropic efficiency characteristic and previously published results [10] at $90 \%$ rotational speed.

flow with axial inflow and neglecting deviation, the rate of change of work coefficient with flow coefficient is

$$
\frac{d \psi}{d \phi}=\tan \left(\kappa_{T E}\right)
$$

so that altering the trailing edge metal angle alters the slope of the rotor work characteristic. What is found, however, is that the camber reduction term produces what appears to be a vertical shift rather than a change in slope. This is due to two separate factors. Only a small range of flow coefficients are considered, all of which are far away from $\phi=0$. Therefore, although the slope is changing, the apparent impact is more the up-down shift of the characteristic over the flow coefficient region of interest. The machine studied in this paper is a transonic compressor and the experimental data shown in Figure 6 (open circles) clearly shows that the characteristic is nonlinear due to compressibility effects as the flow approaches choke; this effect is not captured in the body force model (solid line) because of the lack of metal blockage.

The parallel force coefficients found from calibration are shown in Table 3 and the resultant rotor isentropic efficiency characteristic is shown in Figure 7. At flow coefficients above peak-efficiency, the model is able to capture the efficiency reduction as the flow approaches choke. At flow coefficients below peak-efficiency, the reduction in isentropic efficiency is less severe, matching the performance predicted by the experimental results.

3.2. Example Applications of the Body Force Model. This section examines the body force model's global and local performance metrics in both uniform and nonuniform flow, compared against full-annulus URANS computations. 
TABLE 3: Parallel force coefficient values for stage 67.

\begin{tabular}{lccc}
\hline & Rotor & & Stator \\
\hline$K_{p 1}$ & 0.0145 & $K_{p 1}$ & 0.052 \\
$K_{p 2}$ & 650 & $K_{p 2}$ & 5 \\
$K_{p 2}^{\prime}$ & 1125 & & 0.6199 \\
$M_{r e f}$ & 1.007 & $M_{r e f}$ & \\
$M_{r e f}^{\prime}$ & 0.9870 & & 0.6045 \\
$\left(\bar{M}_{r e l}^{M}\right)_{\text {peak-efficiency }}^{M}$ & 0.9868 & $\left(\bar{M}_{r e l}^{M}\right)_{\text {peak-efficiency }}$ & \\
\hline
\end{tabular}

TABLE 4: Uniform inflow body force model overall performance versus single passage RANS at peak-efficiency flow coefficient.

\begin{tabular}{lccc}
\hline & single passage & body force & $\%$ error \\
\hline$p_{t 2} / p t 1$ (rotor) & 1.48 & 1.49 & 1.43 \\
$\eta_{\text {is }}(\%$, rotor $)$ & 92.5 & 92.4 & 0.03 \\
$\zeta$ (stator) & 0.143 & 0.148 & 3.37 \\
\hline
\end{tabular}

3.2.1. Uniform Flow. To evaluate the model accuracy, a comparison is made against the uniform inflow single-passage RANS calculation. At the peak-efficiency flow coefficient, 0.505, the key performance metrics are shown in Table 4 . The rotor isentropic efficiency is correct to within $0.03 \%$. This degree of accuracy is expected, as the parallel force is calibrated to match the single-passage isentropic efficiency. In the stator, the entropy-based loss coefficient is used to assess accuracy:

$$
\zeta_{3}=\frac{T_{3} \Delta s_{2-3}}{\left(h_{t 3}-h_{3}\right)} .
$$

Here $T_{3}$ is the outlet static temperature, $\Delta s_{2-3}$ is the entropy change across the stator blade row, and $\left(h_{t 3}-h_{3}\right)$ represents the flow of kinetic energy per unit mass at the blade outlet. To calculate the mass-weighted average $\bar{\zeta}^{M}$, streamlines are traced through the stator. Using these streamlines, the local value of $\zeta_{3}$ is calculated for each streamline at a plane of constant axial coordinate near the stator outlet, plane 3 in Figure 3. The error in the loss coefficient is found to be 3.37\% relative to single-passage RANS results.

At flow coefficients away from peak-efficiency, the model is compared against data from Fidalgo et al. [10]. The authors assessed NASA stage 67 with uniform inflow at $90 \%$ of design rotational speed both experimentally and using full-annulus URANS calculations. In Figure 8, the body force model results are plotted against these experimental and CFD results for the rotor isentropic efficiency, total temperature ratio, and total pressure ratio at various flow coefficients. The isentropic efficiency is well matched at flow coefficients both below and above design condition, even outperforming Fidalgo et al. [10] bladed URANS results at flow coefficients below peakefficiency. At the highest flow coefficients investigated, the body force model is unable to capture choking effects. This is expected, as blade metal blockage is not modeled. The total pressure ratio trend is also well-predicted at all flow coefficients, aside from choke conditions, again due to the absence of blockage. There is a systematic increase in total

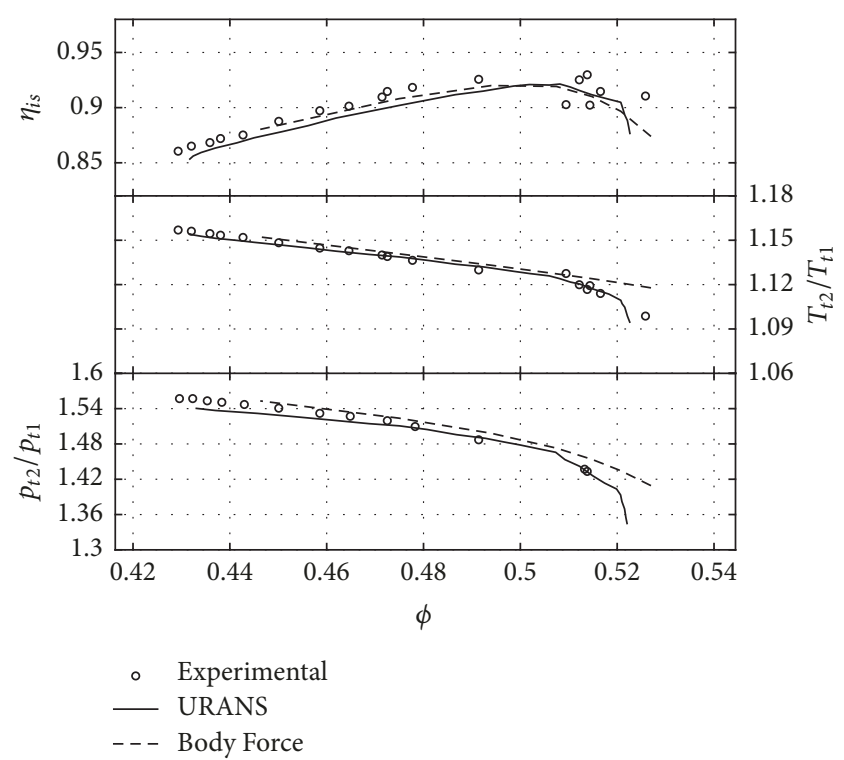

FIGURE 8: Rotor isentropic efficiency, total temperature, and total pressure versus flow coefficient.

pressure ratio relative to the previously published results (between $1.9 \%$ and $4.1 \%$ ). This error stems from various sources: (1) overprediction of rotor work input due to the artificial tip gap work, (2) additional overprediction of rotor work input near choke, and (3) a small error in the rotor isentropic efficiency, especially in its inability to match the severe drop-off near choking. The largest error is expected to be found in the total pressure versus flow coefficient characteristic as it is strongly dependent on the accuracy of both the normal and parallel force models.

In observing the span-wise mass flux profile at rotor trailing edge, shown in Figure 9, the effect of neglecting tip gap leakage is more evident. In the outer $8 \%$ span, the body force model does a poor job in reproducing the desired mass flux distribution. Additionally, slip-wall conditions create another discrepancy in the results in the bottom 5\% span, where the mass flux is overpredicted. At span-wise locations between the two end-walls, the model does an excellent job in capturing the mass flux of the single-passage calculation. Finally, the span-wise work input from the rotor is shown in Figure 10. The error stems from the nature of the normal force modeling approach. Firstly, neglecting the blade lean in 


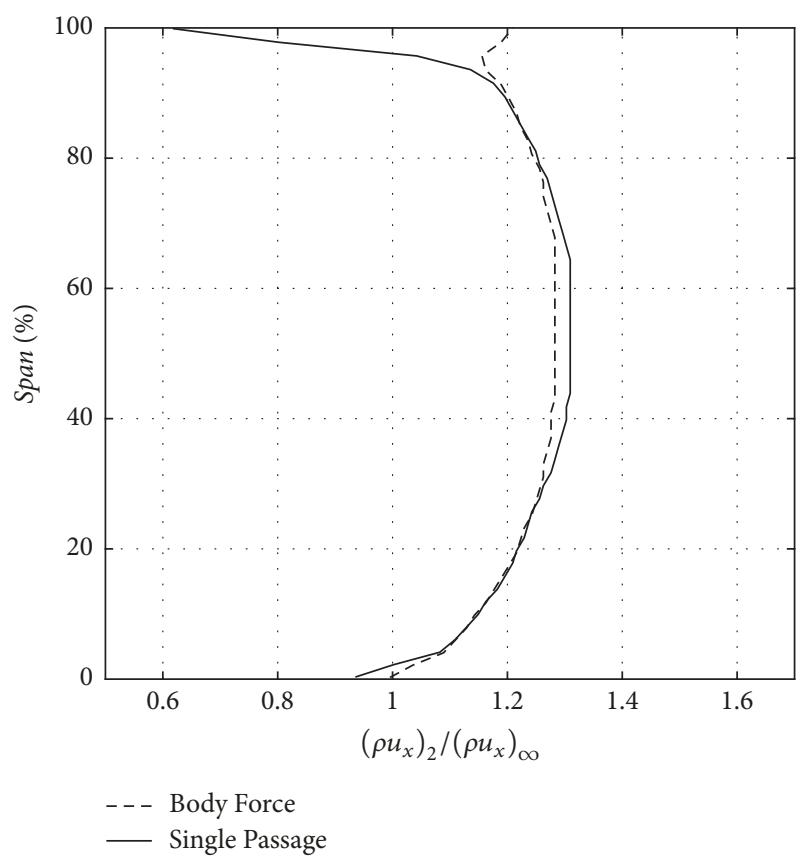

FIGURE 9: Span-wise rotor trailing edge mass flux at peak-efficiency, compared against single-passage results.

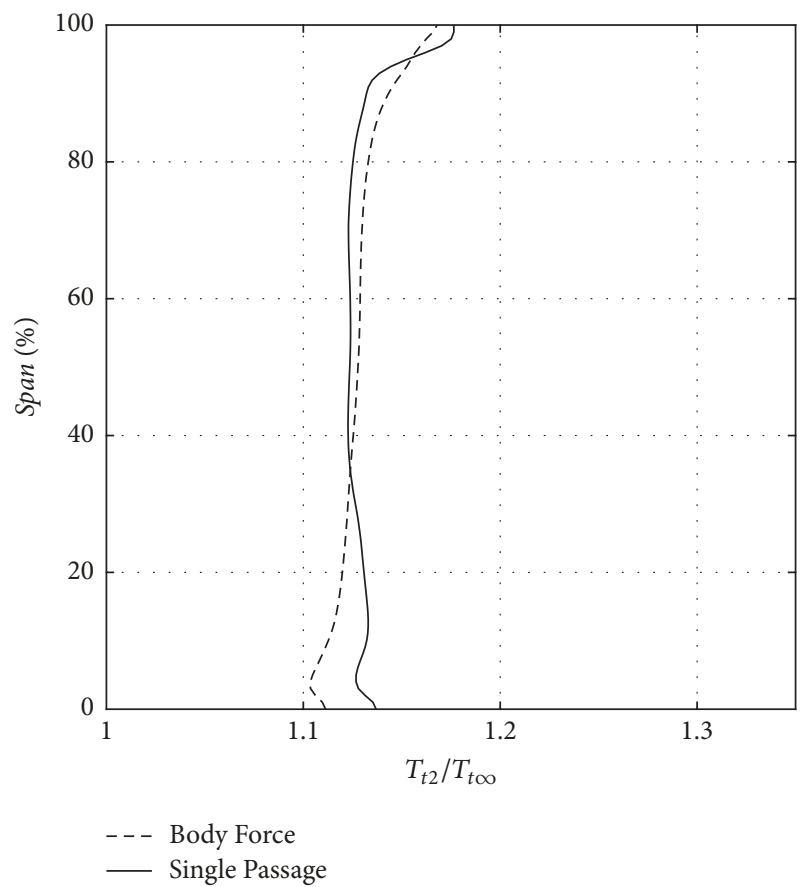

FIGURE 10: Span-wise rotor trailing edge total temperature at peakefficiency, compared against single-passage results.

the normal force calculation may have led to the underprediction near the hub, the area with most significant lean. In addition, the relative flow angle is firstly constrained to match the single-passage results, after which, camber reduction is performed. The method used in this study places emphasis on producing the correct stage work input. As a result, the

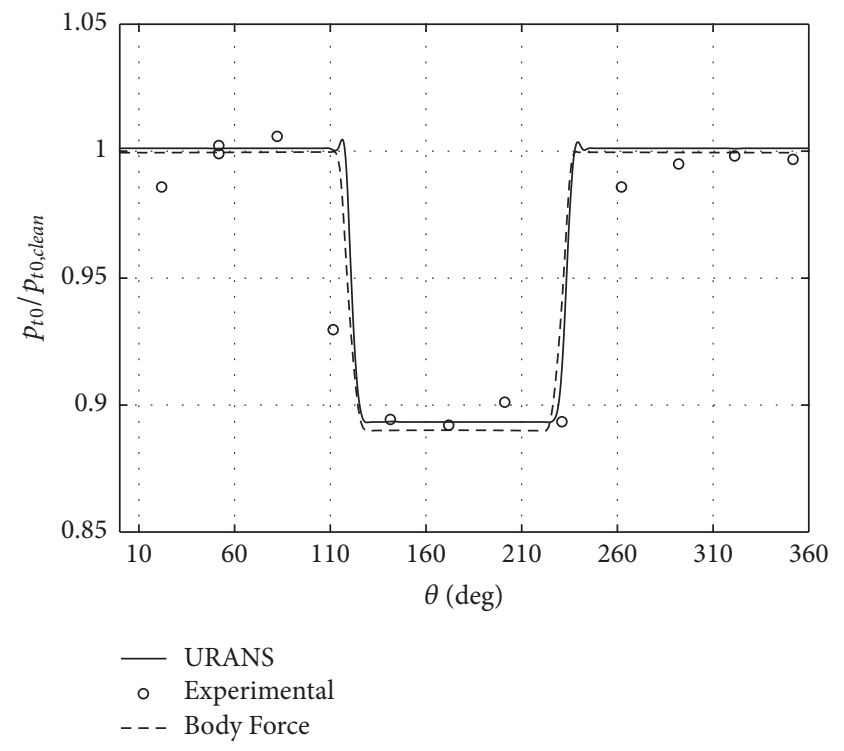

FIGURE 11: Far upstream midspan circumferential traverse of total pressure.

mass-weighted average of total temperature from the body force model compares favorably to that of the single-passage computations. However, the magnitude at each span-wise location leaves room for improvement.

3.2.2. Nonuniform Inflow: $120^{\circ}$ Low Total Pressure Sector. Recall that the intended application of the body force model approach is prediction of compressor response to nonuniform inflow. To assess the model in such conditions, a comparison was made against a nonuniform inflow study performed by Fidalgo et al. [10]. In their work, they subjected NASA stage 67 to a $120^{\circ}$ total pressure distortion. Figure 11 shows the far upstream inlet profile, at station 0 . An operating speed of $90 \%$ of the design rotational speed is again used, with an inlet-corrected flow coefficient based on mass-averaged, total quantities of $\phi=0.49$. The distorted region is set to a total pressure of $0.89 p_{t_{0}, \text { clean }}$ at the domain inlet. The experimental results at inlet involve significant variation from the ideal flow which exists in the CFD. This is due to limitations in how the distortion is produced experimentally given the discontinuous distribution desired. In this sense, the distortion studied is more severe than can be expected to be found in practical applications, such as boundary layer ingestion. For this reason, comparisons in this section will focus on the distributed source term model performance versus URANS results.

The rotor work input and trailing edge absolute swirl angle of the body force model are well matched with URANS results, as shown in the middle part of Figure 12. Both the rotor work input and flow angle follow the URANS trend line near perfectly, while the total pressure ratio trends are captured. The same behavior was observed in uniform flow as discussed earlier. Additionally, the circumferential smearingout of the flow field in the computations with the model 
TABLE 5: Comparison of body force model rotor total pressure and isentropic efficiency changes from clean to distorted inflow versus Fidalgo et al. CFD [10].

\begin{tabular}{lcccc}
\hline & URANS (clean) & URANS (distorted) & body force (clean) & body force (distorted) \\
\hline$p_{t 2} / p t 1$ (rotor) & 1.46 & 1.46 & 1.45 & 1.45 \\
$\eta_{i s}(\%$, rotor $)$ & 93.5 & 92.0 & 91.9 & 90.4 \\
$\Delta \eta_{i s}(\%$, rotor & & -1.50 & & -1.46 \\
\hline
\end{tabular}

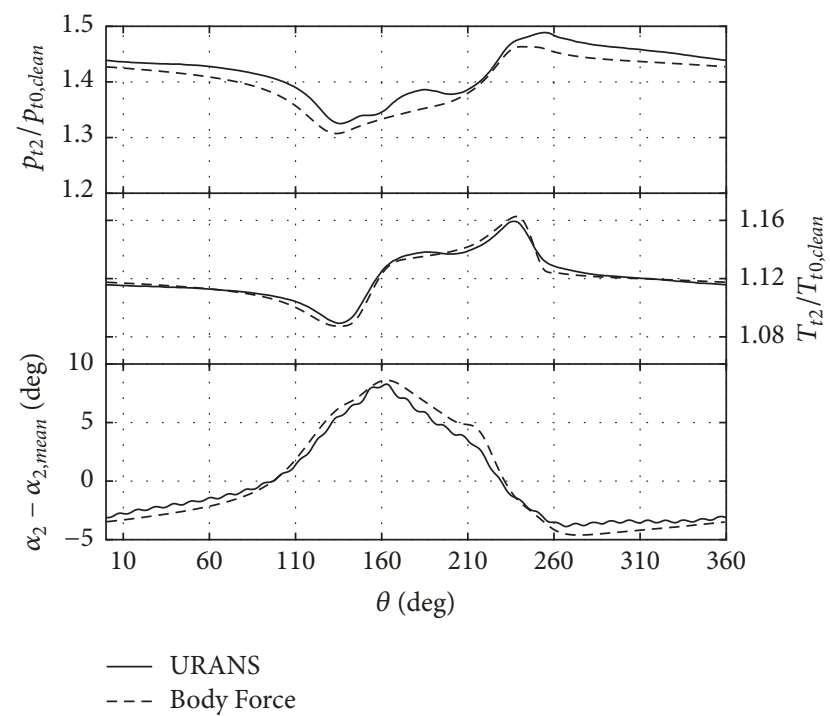

FIGURE 12: Midspan circumferential traverse of absolute whirl angle, total temperature, and total pressure at the rotor trailing edge.

means that the model is unable to capture the swirl variations due to stator upstream influence.

In addition to the circumferential flow characteristics, the body force model also captures blade trailing edge span-wise flow properties. As shown in Figure 13, the total pressure, total temperature, and absolute swirl angles are well matched at a circumferential coordinate location of $73^{\circ}$, a span-wise location just outside of the distortion region; this location is chosen due to the availability of URANS data there. Two notable exceptions are near the hub and tip, for the same reasons discussed earlier (for uniform flow).

Overall performance metrics for the rotor are listed in Table 5. Comparing the results of the URANS calculation by Fidalgo et al. to the performance of the body force model, the change in rotor pressure ratio is perfectly matched to three significant figures (no change between the clean and distorted cases). The change in rotor isentropic efficiency, or the penalty associated with the distortion, is well-captured by the distributed source term model: $-1.50 \%$ in Fidalgo et al. URANS and $-1.46 \%$ in the body force model $(2.67 \%$ error). Given that this is an exaggerated inlet distortion, this degree of accuracy emphasizes the effectiveness of the model for capturing distortion transfer and the response of the turbomachinery to the inflow distortion.

Within the stator blade passage, the qualitative flow response to the distortion is in excellent agreement with the results by Fidalgo et al. [10]. Midspan circumferential

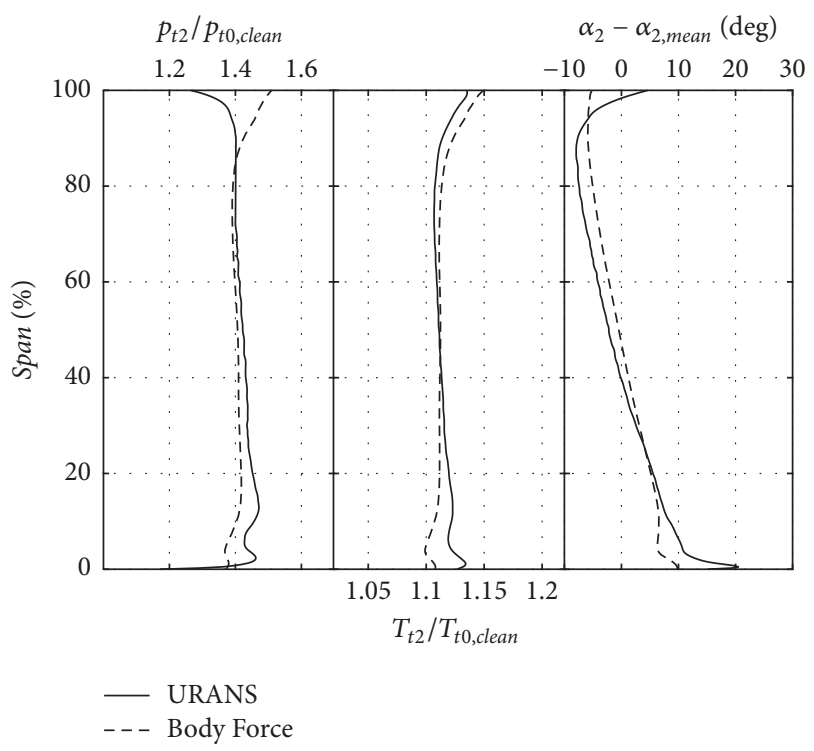

FIgURE 13: Span-wise profiles of total pressure, total temperature, and absolute swirl angle at the rotor trailing edge, $\theta=73^{\circ}$.

traverses of important flow properties downstream of the stator are shown in Figure 14. As expected the stator exhibits an absence of wakes in the source term model. Nevertheless, it is apparent that the flow angles would be in agreement if the blade wakes are filtered out. The total temperature is well-predicted, meaning that the body force model is able to capture the distortion transfer through the stator. The total pressure profile at stator exit is nearly identical to the rotor exit, aside from a vertical shift down, due to excessive stator losses. Circumferential flow redistribution within the stator is minimal as illustrated by the similarity between rotor-exit flow properties and stator exit flow properties. This is expected in a URANS computation, as it has been demonstrated that physical blades block circumferential flow redistribution over length-scales greater than a blade pitch [17].

To quantify the overall stage performance of the body force model in nonuniform inflow, the stator trailing edge total temperature RMS error is $0.31 \%$. The flow field at this location is a result of distortion transfer through both blade rows, showing that distortion transfer and blade response to distortion are well-captured by model. In comparing the total pressure at stator exit of the body force model to the URANS results, an RMS error of 3.1\% exists. This is largely due to excessive loss forces in both the rotor and stator. To isolate the model from this loss force overestimation, an 


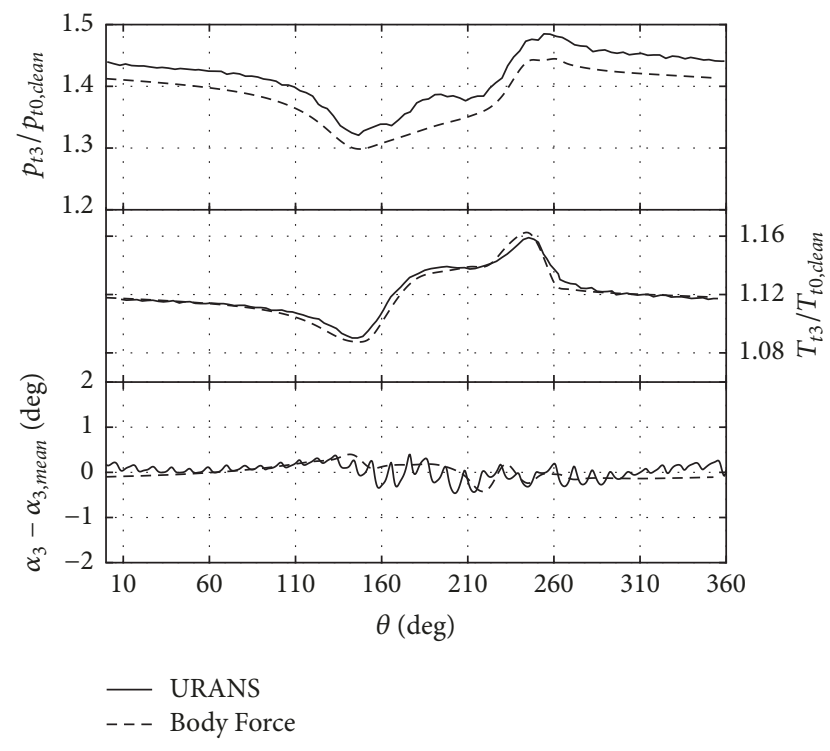

FIGURE 14: Midspan circumferential traverses of absolute swirl angle, total temperature, and total pressure at the stator trailing edge.

alternative RMS error is calculated. Artificially shifting the body force model data such that the mean value is equal to that of the URANS data, an RMS error of $0.57 \%$ is found. This supports the postulation that the model is able to capture distortion transfer as well as blade row losses; the accuracy of the calibration of the loss model is the limiting factor.

\section{Summary and Conclusions}

In this paper, a transonic body force modeling approach is developed by expanding upon existing flow turning and loss models. The model requires calibration of a compressibility correction factor that is both machine and operating speed dependent. Additionally, a camber reduction term is implemented to account for the absence of mass flow blockage. A two-sided loss model is implemented to allow for an enhanced ability to capture choking effects. The resulting model obtained for NASA stage 67 is compared against single-passage uniform inflow and full-annulus nonuniform inflow.

The key outcomes from this work are the following: (1) a normal force compressibility correction in combination with camber modification is successful in producing a model which captures span-wise work input; (2) the body force model is able to capture global performance metrics (rotor work and isentropic efficiency) in both uniform and nonuniform inflow; (3) the body force model is able to capture localized flow properties and flow redistribution in both uniform and nonuniform inflow; and (4) the modeling approach used is expected to be robust, since there are no limitations to blade geometry, flow regime, or incoming flow properties.

At peak-efficiency, in uniform flow, the body force model is able to predict the rotor total pressure ratio within $1.43 \%$ as well as the isentropic efficiency within $0.03 \%$. In the stator domain, the entropy-based loss coefficient is found to have an error of $3.37 \%$. Considering the rotor total temperature ratio, total pressure ratio, and isentropic efficiency away from peak-efficiency, the largest error is found within the total pressure ratio. Even still, the overestimation in total pressure ratio is limited to $4.1 \%$ compared to URANS results. In the case of a severe nonuniform inflow, the stator trailing edge total temperature ratio at midspan has an RMS error of $0.31 \%$, showing that the distortion transfer and blade work input are well-predicted throughout the entire stage.

Future work will involve an automated, optimized scheme to calibrate the normal and parallel force models with greatly reduced user effort. Additionally, a mass flow blockage source term could be implemented to allow for the removal of the $\Delta \kappa$ term and for more accurate blade loading. The model obtained is useful for studying the various effects of nonuniform flows on transonic compressors. Most importantly, the reduced computational cost when compared to full-annulus URANS allows for many different distortions to be studied. The intended application for this model is the study of efficiency scaling associated with varying distortion patterns and intensities.

\section{Nomenclature}

$\begin{array}{ll}A: & \text { Reference area } \\ b: & \text { Staggered blade spacing } \\ B: & \text { Number of blades } \\ c_{l}: & \text { Lift coefficient per unit span } \\ c_{x}: & \text { Blade axial chord } \\ f: & \text { Volumetric source term per unit mass } \\ g_{r e d}: & \text { Local rotor reduced frequency } \\ h: & \text { Enthalpy } \\ K_{n}: & \text { Blade loading coefficient } \\ \widehat{K}_{p}: & \text { Combined loss coefficient } \\ K_{p 1}: & \text { Design loss coefficient } \\ K_{p 2}: & \text { Off-design loss coefficient } \\ \dot{m}: & \text { Corrected mass flow rate } \\ M_{r e l}: & \text { Relative Mach number } \\ M_{r e f}: & \text { Reference Mach number } \\ \widehat{n}: & \text { Camber surface normal } \\ p: & \text { Pressure } \\ r: & \text { Radial coordinate } \\ s: & \text { Entropy } \\ T: & \text { Temperature } \\ v: & \text { Velocity component in stationary frame } \\ V: & \text { Velocity magnitude in stationary frame } \\ W: & \text { Velocity magnitude in rotating frame } \\ x, y, z: & \text { Cartesian coordinates } \\ \alpha: & \text { Absolute swirl angle } \\ \beta: & \text { Relative swirl angle } \\ \delta: & \text { Flow deviation } \\ \epsilon: & \text { Compressibility correction } \\ \zeta: & \text { Entropy-based loss coefficient } \\ \eta: & \text { Isentropic efficiency } \\ \theta: & \text { Circumferential coordinate } \\ \kappa: & \text { Local blade camber angle } \\ \Lambda: & \text { Camber reduction constant } \\ \rho: & \text { Density } \\ & \end{array}$


$\sigma$ : Blade solidity

$\phi$ : Flow coefficient

$\psi$ : Work coefficient

$\omega$ : Corrected rotational speed.

\author{
Subscripts \\ 0-4: Axial measurement planes \\ in: Inlet \\ is: Isentropic \\ $L E$ : Leading edge \\ $n$ : $\quad$ Normal to stream-wise direction \\ p: Parallel to stream-wise direction \\ $t$ : Total/stagnation quantity \\ TE: Trailing edge \\ $\infty$ : Free-stream.
}

\section{Superscripts}

M: Mass-averaged quantity.

\section{Data Availability}

The data used to support the findings of this study are available from the corresponding author upon request.

\section{Conflicts of Interest}

The authors declare that they have no conflicts of interest regarding this publication of this article.

\section{Acknowledgments}

This work is funded by the NSERC Discovery Grants program. It is based upon the work completed in Hill's thesis [18]. Computational resources are provided by the facilities of the Shared Hierarchical Academic Research Computing Network (SHARCNET: www.sharcnet.ca) and Compute/Calcul Canada. The authors would like to thank Dr. Ewan Gunn and Dr. Vincente Fidalgo for the stage 67 stator model used in this work.

\section{References}

[1] J. J. Defoe, M. Etemadi, and D. K. Hall, "Fan performance scaling with inlet distortions," Journal of Turbomachinery, vol. 140, no. 7, 11 pages, 2018.

[2] J. J. Defoe and Z. S. Spakovszky, "Effects of boundary-layer ingestion on the aero-acoustics of transonic fan rotors," Journal of Turbomachinery, vol. 135, no. 3, 2013.

[3] J. J. Defoe and Z. S. Spakovszky, "Shock Propagation and MPT Noise From a Transonic Rotor in Nonuniform Flow," Journal of Turbomachinery, vol. 135, no. 1, 2012.

[4] W. Thollet, G. Dufour, X. Carbonneau, and F. Blanc, "Bodyforce modeling for aerodynamic analysis of air intake - fan interactions," International Journal of Numerical Methods for Heat \& Fluid Flow, vol. 26, no. 7, pp. 2048-2065, 2016.

[5] J. Guo and J. Hu, "Development of body force model for steady inlet distortions in high-speed multistage compressor," Proceedings of the Institution of Mechanical Engineers, Part G:
Journal of Aerospace Engineering, vol. 231, no. 9, pp. 1650-1659, 2017.

[6] F. Marble, Three-Dimensional Flow in Turbomachines. High Speed Aerodynamics and Jet Propulsion, 10, 83-166, 1964.

[7] Y. Gong, C. S. Tan, K. A. Gordon, and E. M. Greitzer, "A computational model for short-wavelength stall inception and development in multistage compressors," Journal of Turbomachinery, vol. 121, no. 4, pp. 726-734, 1999.

[8] A. Peters, Z. S. Spakovszky, W. K. Lord, and B. Rose, "Ultrashort nacelles for low fan pressure ratio propulsors," Journal of Turbomachinery, vol. 137, no. 2, 2015.

[9] D. K. Hall, E. M. Greitzer, and C. S. Tan, "Analysis of fan stage conceptual design attributes for boundary layer ingestion," Journal of Turbomachinery, vol. 139, no. 7, 2017.

[10] V. J. Fidalgo, C. A. Hall, and Y. Colin, "A Study of FanDistortion Interaction Within the NASA Rotor 67 Transonic Stage," Journal of Turbomachinery, vol. 134, no. 5, 2012.

[11] A. Strazisar, J. Wood, M. Hathaway, and K. Suder, Laser Anemometer Measurements in a Transonic Axial-Flow Fan Rotor, 1989.

[12] K. Patel, C. Novak, and J. Defoe, "Novel numerical approach for generation and propagation of rotor-stator interaction noise," in Proceedings of the 22nd AIAA/CEAS Aeroacoustics Conference, 2016, France, June 2016.

[13] ANSYS CFX Solver Theory Guide, Canonsburg, PA, USA, 2013.

[14] Pointwise User's Manual, 4th edition, 2016.

[15] ANSYS TurboGrid User's Guide, Canonsburg, PA, USA, 2013.

[16] F. Menter, "Zonal Two Equation k-w Turbulence Models For Aerodynamic Flows," in Proceedings of the 23rd Fluid Dynamics, Plasmadynamics, and Lasers Conference, Orlando, FL, U.S.A..

[17] E. J. Gunn and C. A. Hall, "Aerodynamics of Boundary Layer Ingesting Fans," in Proceedings of the ASME Turbo Expo 2014: Turbine Technical Conference and Exposition, p. V01AT01A024, Düsseldorf, Germany.

[18] D. Hill, Compressor Performance Scaling in the Presence of NonUniform Flow, University of Windsor, Canada, 2017. 


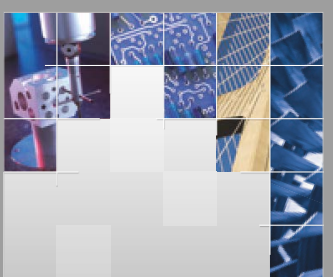

\section{Enfincering}
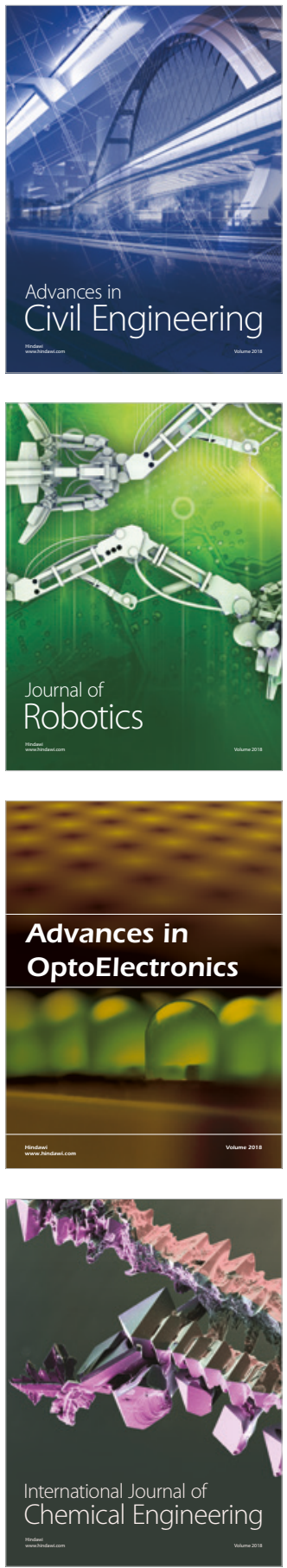

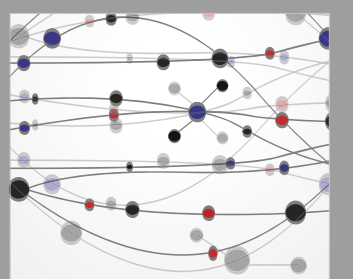

\section{Rotating \\ Machinery}

The Scientific World Journal

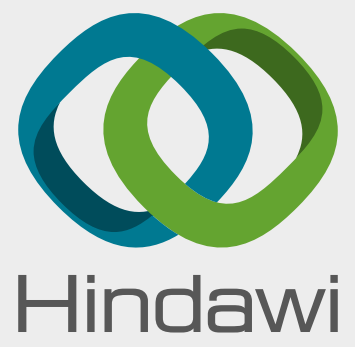

Submit your manuscripts at

www.hindawi.com
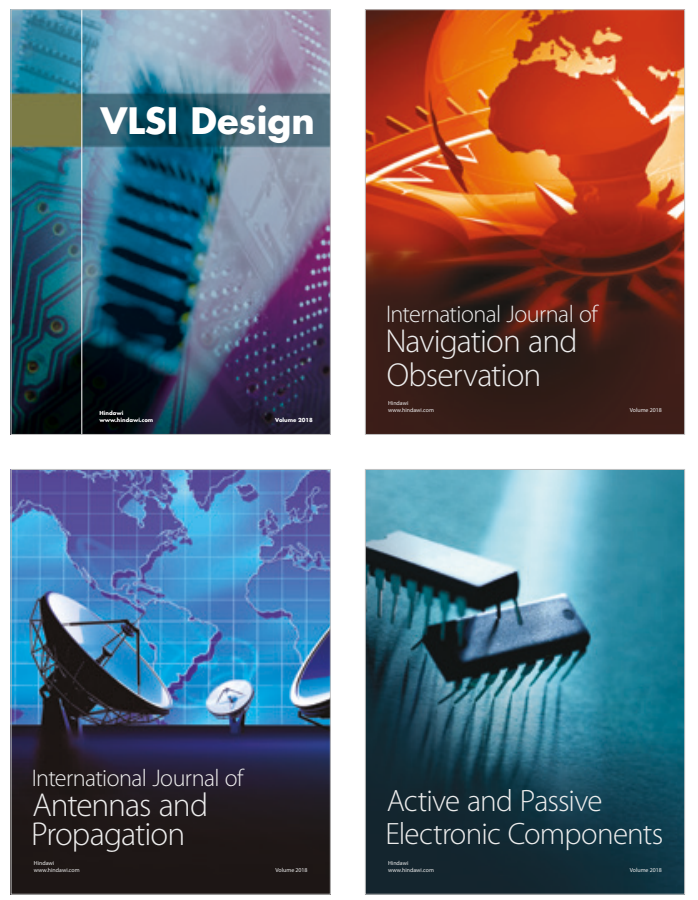
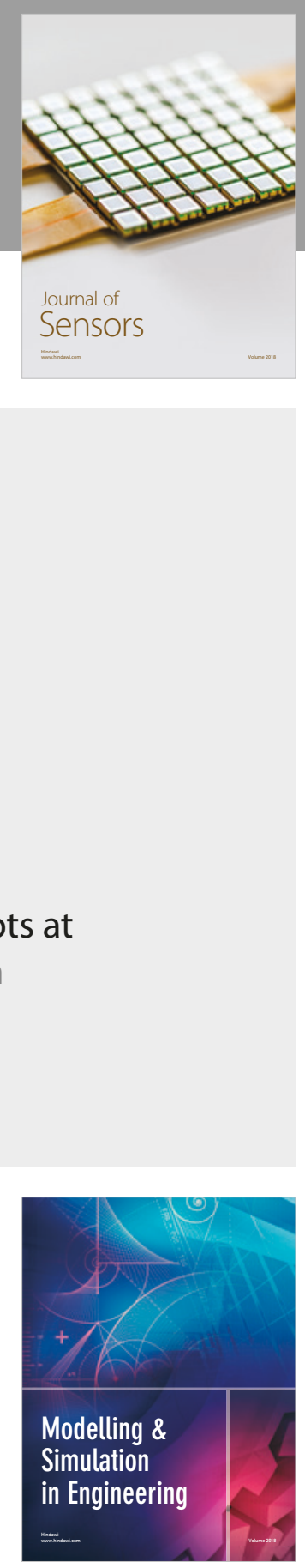

\section{Advances \\ Multimedia}
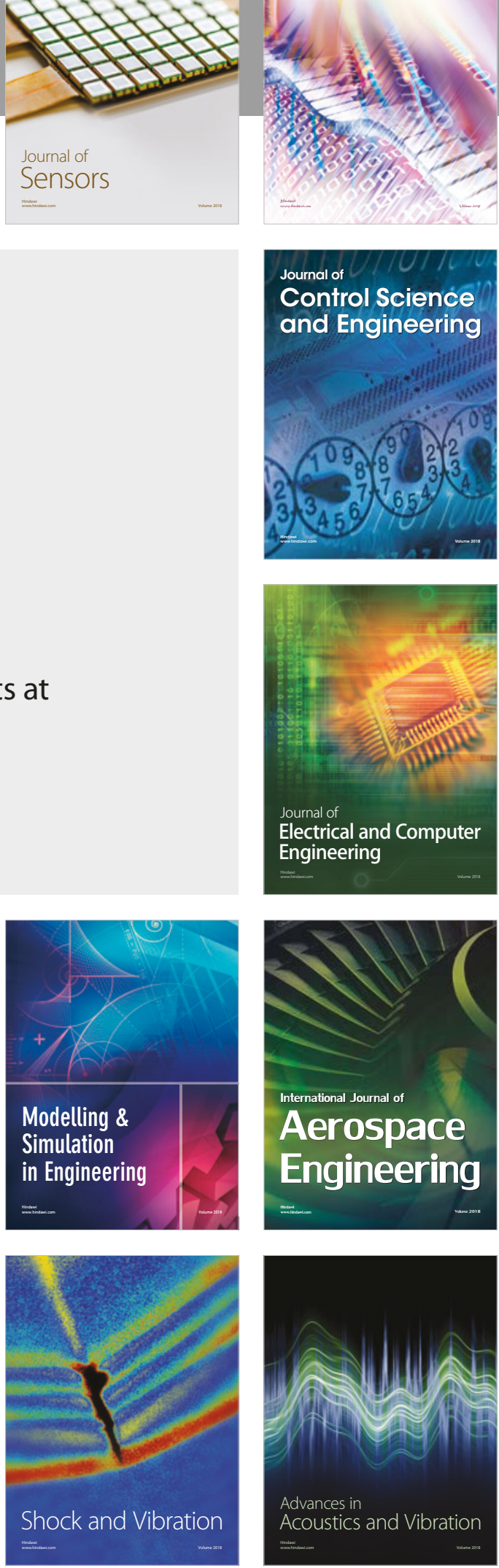